\title{
HISTORIA ORAL Y ANÁLISIS NARRATIVO DE NARRATIVAS EN LA INVESTIGACIÓN EN LA EDUCACIÓN MATEMÁTICA BRASILEÑA
}

\section{Oral History and narrative analysis of narratives in the mathematics education research in Brazil}

\author{
Fernando Guedes CuRY \\ Universidade Federal do Rio Grande do Norte/Brasil \\ Correo-e: matfernando@yahoo.com.br
}

Recepción: 20 de diciembre de 20I6. Envío a informantes: ro de marzo de 2017.

Aceptación definitiva: ro de junio de 2017

Resumen: En este artículo presentamos la Historia Oral como una alternativa para las investigaciones en historia de la educación matemática a partir de algunas experiencias realizadas en Brasil. Hacemos una discusión de lo que significa la Historia Oral y cómo se puede llevar a cabo resaltando sus posibilidades y limitaciones. Considerándola como metodología de investigación cualitativa, actualmente se usa en diversas investigaciones historiográficas o no. Además de sus posibilidades, también presentamos algunos ejemplos de trabajos que se han desarrollado a lo largo de la última década con énfasis en aquellos que han usado el análisis narrativo de narrativas.

Palabras clave: Historia Oral; metodología de investigación; historia de la educación matemática; análisis narrativo.

AвSTRACT: This paper presents the Oral History as an alternative for the research on the history of mathematics education according to some experiences performed in Brazil. We discuss how Oral History can be comprehended and how it can be developed by highlighting some of its possibilities and limitations. As a qualitative research methodology, the Oral History has been used either in historiographics' researches studies, or not, adopting different theoretical perspectives. In 
this way, we present some examples of academic works that were done in the last decade with emphasis on the use of narrative analysis of narratives as an alternative for their data analyzes.

KEY WORDS: Oral History; research methodology; history of mathematics Education; narrative analysis.

\section{Introducción: nuestras concepciones sobre la historia oral}

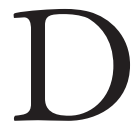

ESDE LAS NUEVAS PROPUESTAS EN EL SIGLO PASADO para la historia que buscaban la ampliación de perspectivas como un movimiento derivado del análisis de nuevos enfoques o la expansión de fuentes, la Historia Oral surge como una alternativa viable, es decir, escribir historias a partir de la experiencia acumulada por una persona y apoyadas preferiblemente en documentos escritos. Está asentada en una opción diferenciada e interesante del mundo, una visión más amplia de la historia, que sospecha de las metanarrativas (eurocéntricas, científicas, teológicas, o que consideran la historia como lineal y progresiva), adoptando, por el contrario, la interdisciplinariedad y la noción de historia-problema, posibilitando diferentes lecturas de historias variables, aceptando «verdades plurales», que coexisten, versiones en lugar de «una verdad histórica».

Muchos estudiosos de este campo ${ }^{\mathrm{I}}$ sugieren que la Historia Oral (en adelante HO) surgió en los Estados Unidos poco después de la Segunda Guerra Mundial, cuando en diferentes estudios se usaron métodos que incluían entrevistas e historias de vida y que obtuvieron importantes resultados con respecto a problemas sociales urbanos. En ese país también se fundó la Asociación de Historia Oral que, en I948, estableció la Ho como una técnica de documentación histórica. Esa versión de la Ho persistió durante mucho tiempo y sólo en la década de 1970 resurgió al interesarse en ciertas historias especialmente de las minorías o grupos marginados.

En los años 1990, la Ho en Brasil pasó a tener mayor visibilidad en congresos y discusiones entre los historiadores brasileños y extranjeros, principalmente a partir de la creación de la Assossiação Brasileira de História Oral, que favoreció que los investigadores se especializasen en el tema. El investigador José Carlos Sebe Bom Meihy explica que la Ho tardó en establecerse en Brasil debido a la falta de tradiciones académicas institucionales en el desarrollo de proyectos que registraran historias locales en el país y esto, a su vez, debido a la ausencia de vínculos de las universidades con la cultura popular.

El trabajo con Ho se basa fundamentalmente en la memoria. Actualmente, el concepto y el funcionamiento de la memoria tienen contribuciones de las ciencias

I Thompson, P.: A voz do passado: história oral, São Paulo, Paz e Terra, i998. Meiny, J. C. S. B.: Manual de História Oral, São Paulo, Loyola, 2005. Alberti, V.: «Narrativas pregnantes como jogos de linguagem: possibilidades da história oral à luz da teoria da linguagem de Wittgenstein», História Oral, Rio de Janeiro, v. II (2008), pp. I27-I48. 
HISTORIA ORAL Y ANÁLISIS NARRATIVO DE NARRATIVAS EN LA INVESTIGACIÓN EN LA EDUCACIÓN MATEMÁTICA BRASILEÑA FERNANDO GUEDES CURY

físicas y biológicas. Además de la psicología y de las ciencias sociales que miran la memoria individual y colectiva como uno de sus focos de investigación. Estos estudios involucran los conceptos de retención, recuerdo, olvido, selección, resentimiento, euforia y entusiasmo. Como resultado de otros numerosos estímulos, la memoria debe ser entendida como una construcción hecha en tiempo presente de vivencias que ocurrieron en el pasado.

Pasado el período de consolidación, la Ho se usa en la investigación y en el desarrollo de las ciencias sociales, la enfermería, la antropología y los estudios culturales. Debido a esta amplia variedad de aplicaciones, ha permitido lograr diferentes formas de comprensión, entre las que se describen claramente tres posiciones principales: una «técnica», un «método» y una «metodología».

Los que abogan por la Ho como una técnica están interesados únicamente en la creación y conservación de archivos orales, incluyendo aquellos que utilizan las entrevistas en cualquiera de sus formas, por ejemplo, para complementar otros tipos de fuentes, en general, escritas. Para los seguidores de esta línea lo que importa son las grabaciones, las transcripciones y entrevistas y el aparato que la guía (formas de transcripción de grabaciones, modelos de organización de colección y otros). A pesar de ello, muchos de los que han trabajado bajo este punto de vista no siempre defienden conscientemente tal "postura técnica», esta opción es el resultado de la relación que algunos eruditos parecen mantener con el Ho: verla como una utilidad para satisfacer las necesidades de la investigación o de sus deberes profesionales.

Los que consideran la Ho como una disciplina mantienen que gracias a ella se han ampliado las técnicas específicas de investigación, los procedimientos metodológicos y los conceptos asociados ${ }^{2}$. Estos autores señalan algunos de los conceptos que representan perspectivas específicas y temas reconocidos a partir de Ho, incluso por aquellos que no le dieron el estatus de técnica:

- El testimonio oral presenta el núcleo de la investigación, nunca su parte accesoria.

- El uso sistemático de testimonios orales permite aclarar trayectorias individuales, eventos o procesos que algunas veces no pueden ser entendidos de otra manera.

- En Ho hay generación de documentos (las entrevistas) que tienen una característica única: son el resultado del diálogo entre entrevistador y entrevistado, entre sujeto y objeto de estudio.

- El estudio de fuentes orales está basado en los puntos de vista individuales, expresados en las entrevistas.

- La Ho permite la reflexión histórica de la historia de la actualidad.

- En ese tipo de estudio el objeto es recuperado y reconstruido por la memoria de los sujetos.

- La ho es ampliamente practicada fuera del mundo académico, entre los grupos y comunidades interesadas en recuperar y reconstruir su propia memoria

Amado, J. y Ferreira, M. M.: Usos e Abusos da Historia Oral, Rio de Janeiro, Fundação Getúlio Vargas, 1996. 
y eso ha elevado las tensiones (y reflexiones), desde las perspectivas, de los objetos y del funcionamiento académico.

- Tanto la narrativa como la forma de construcción y organización del discurso son valorados por el historiador.

Estas ideas también son compartidas por los que consideran la Ho como metodología. Sin embargo, lo que los distingue del grupo anterior es que bajo esta perspectiva se reconoce en la HO un área de estudio e investigaciones con objetos propios y capacidad para proporcionar, por sí misma, soluciones teóricas a los problemas que se presentan en la práctica, por ejemplo, temas como los conflictos e integraciones entre historia y memoria, entre sujeto y objeto de estudio, entre historia, biografía y autobiografía, y entre los usos sociales del discurso3.

Una tercera postura sobre Ho, entendida como metodología, establece y ordena los procedimientos de trabajo -como diferentes tipos de entrevistas y las implicaciones de cada una para la investigación, las distintas posibilidades de transcripción de las declaraciones, sus ventajas e inconvenientes, las diferentes maneras en que el investigador relaciona sus respuestas y las influencias en su trabajo-, siendo un enlace que articula la teoría y la práctica de trabajo. Además, concebir la Ho como una metodología de investigación cualitativa es reconocer su carácter único y es incompatible con las generalizaciones. En esta línea, el trabajo con Ho dedicado a recopilar testimonios de personas acerca de los procesos históricos y sociales cuenta con otras varias posibilidades metodológicas y cognitivas como, por ejemplo, revelar nuevos campos y temas de investigación; presentar nuevas versiones de los hechos ya conocidos; recuperar memorias individuales y comunitarias; rescatar información acerca de eventos no registrados; complementar los registros ya existentes; y permitir la asociación de eventos de la vida pública y de la vida privada a través de narrativas individuales. Así, la construcción intencional de las fuentes históricas propuestas por estudios que utilizan la Ho ocurre a través de la exploración de la oralidad, narraciones que se forman a partir de esta oralidad, es decir, de las situaciones de entrevista4.

Por otra parte, reconocemos que la Ho tiene peculiaridades que son como retos para los oralistass. Algunos de ellos son: sólo es aplicable a tiempos contemporáneos; predomina la subjetividad que debe ser controlada desde el momento de la realización de las entrevistas hasta su tratamiento y análisis; la responsabilidad ética del investigador con los sujetos y con el material grabado; la dificultad de exponer las expresiones gestuales y faciales de los entrevistados en las obras que se publican generalmente en forma escrita; la complejidad de tratar con la memoria y, por tanto, con el olvido. Sin embargo, estos límites no ocultan ni descalifican la riqueza informativa o interpretativa que, es habitual, atrae a quienes trabajan con Ho.

Amado, J. y Ferreira, M. M.: Usos e Abusos da Historia Oral, Rio de Janeiro, Fundação Getúlio Vargas, 1996.

4 Thompson, P.: A voz do passado: história oral, São Paulo, Paz e Terra, 1998.

Palabra usada para indicar a los que hacen uso de la Historia Oral en la investigación. 
Cabe también destacar algunos conceptos erróneos, que deben evitarse y que todavía se producen cuando se habla de Ho. Uno de ellos es considerar que un relato resultante de una entrevista ya es la historia que se desea contar. Si lo fuera, estaríamos confundiendo la memoria (cuyo registro de préstamos de la oralidad genera las fuentes defendidas como legítimas por los oralistas) con la Historia (o más bien la historiografía, resultado específico de una hermenéutica de las fuentes ya disponibles o creadas) ${ }^{6}$. Es decir, como veremos con más detalle a continuación, la entrevista puede considerarse como una fuente que necesita de interpretación y no es una revelación del mundo real por «sí misma».

Otra idea falsa está en la utilización de expresiones tales como «historia democrática» o «historia desde abajo» para resaltar la labor relacionada con esta metodología. Por consiguiente, es incorrecto acusar de no democráticos a los investigadores que entrevistan a los miembros de una élite, diciendo que han estudiado visiones del mundo y de la vida de una «historia vista desde arriba». La autora añade que este tipo de polarización debilita la especificidad de la Ho no permitiendo el registro y estudio de la experiencia de un creciente número de personas, independientemente de su posición social.

[...] a ideia de 'dar voz' às minorias, tão cara aos pesquisadores 'militantes', acaba reforçando as diferenças sociais: é o pesquisador que concede aos 'de baixo' a possibilidade de se expressarem, pois eles são incapazes de fazê-lo por si sós! [...] Até que ponto os estudos sobre grupos sociais marginalizados respondem a uma demanda desses mesmos grupos? Quem lê suas entrevistas? Como fazer que o resultado da pesquisa retorne ao grupo investigado? Em vez de imaginar que está imbuído em uma 'missão democrática', o pesquisador deve reconhecer que a necessidade de ouvir os 'de baixo' parte, antes de mais nada, dele mesmo, da instituição em que trabalha, do órgão que financia sua pesquisa, desde que esteja consciente disso e de suas implicações de sua decisão, é claro que nada impede que se lance à pesquisa’ .

Por lo tanto, la Ho como metodología de investigación debe superar dualidades de tipo ganadores/perdedores, global/local, académico/popular y, por supuesto, oral/escrito, para establecerse en sintonía con la investigación actual en las ciencias sociales que reconocen las múltiples influencias e interpretaciones del mundo.

Pero, a pesar de que la Ho no está exclusivamente vinculada a los estudios y temas históricos, no debemos apartarla mucho de estas cuestiones y, por lo tanto, no sería apropiado ejercitar la Ho desconectada de una concepción historiográfica que apoye tales acciones. La justificación, según los autores mencionados, es la siguiente: quien utiliza la Ho para la comprensión de alguna cosa estará, intencionalmente, produciendo fuentes (históricas) que pueden servir para

6 Garnica, A. V. M.; Fernandes, D. N. y Silva, H.: «Entre a amnésia e a vontade de nada esquecer: notas sobre Regimes de Historicidade e História Oral», Bolema, Rio Claro, v. 25 (20II), pp. $213-250$.

Alberti, V.: «Narrativas pregnantes como jogos de linguagem: possibilidades da história oral à luz da teoria da linguagem de Wittgenstein», História Oral, Rio de Janeiro, v. II (2008), pp. I27-I48. 
exponer las perspectivas biográficas y contextuales no sólo en lo que se estudia, sino sobre quienes, con sus declaraciones, nos permitirán una aproximación al objeto estudiado.

\section{Concepciones, apropiaciones y trabajo de campo}

Si hace unos diez años la Historia Oral parecía escasa en investigaciones sobre las prácticas educativas en España -a pesar de su reconocido potencial para cuando nos referimos a etapas próximas en el tiempo $-{ }^{8}$ las investigaciones con la HO y las prácticas de investigación desarrolladas a partir de los años 2000 en Brasil, por los investigadores que conforman el Grupo de Historia Oral y Educación Matemática (GHOEM) 9 y otros grupos más recientes (HMEP ${ }^{10}$ y GPEP $^{\mathrm{II}}$ ), destacan, por lo menos, dos características principales de esta metodología. La primera concierne al análisis de datos y los procedimientos de investigación: las investigaciones se centran en la colección de testimonios grabados de personas involucradas, directa o indirectamente, con la educación matemática, en atención a los temas de cada investigación. La segunda, una propuesta aún en desarrollo, cuyo objetivo es diseñar un mapa (histórico) de la formación del profesorado de matemáticas en Brasil y de los actores involucrados en este escenario, al mismo tiempo que se estudian y evalúan los recursos metodológicos empleados. Este proyecto tiene algunas características fundamentales: (a) la larga duración (en el sentido habitual del término, puesto que el proyecto se inició a principios de 2000 y no hay ninguna predicción de cierre); (b) considera una variedad de espacios geográficos y culturales, porque sus subproyectos pretenden tejer narrativas acerca de la formación y la actividad de los maestros de matemáticas de diferentes regiones brasileñas y de diferentes grupos socioculturales; (c) decide centrarse en diversos momentos de la historia de la educación y de la educación matemática (con énfasis en la «actualidad», pues es la opción central -aunque no exclusiva- de la Ho); (d) estudia y adopta diversas técnicas de composición narrativa; (e) analiza varias caras del proceso educativo (estudia los libros de texto, las prácticas y desempeño de los docentes, las formas de apropiación o rechazo de políticas públicas de la organización escolar, espacios arquitectónicos y materiales escolares, entre otros); (f) hace uso de múltiples fuentes; y $(\mathrm{g})$ aún cuenta con la participación en el proyecto de investigadores

8 Hernández Díaz, J. M.: «Recensión: Vidigal, L.: "Os testemunhos oráis na escola. Historia oral e projectos pedagógicos”, Porto, Ediçoes Asa, I996», História de la Educación, vol. I6 (1997), pp. 647-648.

9 Página web de los grupos de investigación del Consejo Nacional de Investigación de Brasil: http://DGP.CNPq.br/DGP/espelhogrupo/oor8479156055834.

ro Página web del Grupo Historia de la Educación Matemática en Investigación. http://DGP. CNPq.br/DGP/espelhogrupo/3978760600539715.

" Página web del Grupo de Estudios e Investigación en Historia de la Educación Matemáticas del Estado do Rio Grande do Norte/Brasil. http://DGP.CNPq.br/DGP/espelhogrupo/9562255289198995. 
HISTORIA ORAL Y ANÁLISIS NARRATIVO DE NARRATIVAS EN LA INVESTIGACIÓN EN LA EDUCACIÓN MATEMÁTICA BRASILEÑA FERNANDO GUEDES CURY

en diferentes niveles de formación: de graduación (Licenciatura), máster, doctorado y postdoctorado ${ }^{12}$.

El mapeo histórico de la formación y de la actuación de los maestros de matemáticas en Brasil es -y siempre será-, por lo tanto, un proyecto en curso, una iniciativa sin terminar. En el estado de São Paulo se han hecho estudios desde la regiones de Santos (litoral) hasta el extremo oeste sobre la formación de los maestros de matemáticas en escuelas rurales y sobre las escuelas agrícolas paulistas, por ejemplo; también hay estudios sobre la formación de grupos de investigadores en educación matemática, específicamente en el Centro de Educación Matemática (CEM) de la ciudad de São Paulo, formado a partir de un núcleo vinculado al movimiento de las matemáticas modernas. En el estado de Paraná hay investigaciones sobre prácticas educativas actuales de las instituciones privadas de educación universitaria en el interior del estado y sobre el grupo NEDEM (Centro de Estudio y Difusión de la Enseñanza de las Matemáticas) responsable de poner en práctica la matemática moderna allí. En el estado de Santa Catarina, se desarrolló un estudio sobre las escuelas alemanas de la región de Blumenau y en Mato Grosso se investigó la formación y el desempeño de los maestros. En el nordeste brasileño, en Paraíba y Maranhão, respectivamente, han estudiado el desempeño docente y la formación matemática en los estados. Hay también estudios sobre las concepciones sobre las matemáticas y su enseñanza de maestros cuando elegían libros didácticos para sus clases sobre cómo, sociológicamente, los maestros se convirtieron en los profesionales que son actualmente; sobre la Campaña de Mejoramiento y Difusión de la Educación Secundaria (CADES), y sobre los cursos de educación a distancia (en concreto, el Proyecto Minerva, de la educación por la radio). Se han desarrollado muchos otros trabajos y se seguirá progresando en esta línea buscando siempre el diálogo con las fuentes de diversa índole (escritas, gráficas, fílmicas, etc.), revelando las fuentes orales y la pluralidad de perspectivas (interpretaciones) $)^{13}$.

En la práctica, las investigaciones comienzan, por supuesto, y tales como estudios hechos en España ${ }^{14}$, con el diseño de un proyecto de investigación que deberá proporcionar una base teórica para fundamentar el estudio y defender su relevancia, así como señalar su posible impacto en la sociedad o en el campo de estudios a la que pertenece. Además, hay que aclarar los objetivos del proyecto y establecer, aunque de manera preliminar, la red de personas para las entrevistas -lo que indica, por ejemplo, su exigibilidad-. Los proyectos también pueden recurrir al menos a dos modos básicos de comprensión de la actualidad: la Historia de Vida y la Historia Oral Temática. En el primer caso, el investigador está interesado por uno o más declarantes seleccionados y se investiga su vida como

12 Garnica, A. V. M.; Fernandes, D. N. y Silva, H.: «Entre a amnésia e a vontade de nada esquecer: notas sobre Regimes de Historicidade e História Oral», Bolema, Rio Claro, v. 25 (20II) pp. 213-250.

${ }_{13}$ Para ver una copia digital de estos trabajos, visitar la página web del GHOEM: www.ghoe.com.

I4 Marqués, S.: «Importancia de la investigación oral para el estudio de la historia de la escuela franquista", Historia de la Educación, v. I2-13 (1993-1994), pp. 435-447. 
un todo, es decir, el declarante se narra a sí mismo. En el segundo modo, el trabajo con la Historia Oral temática cuenta con testimonios orales recogidos de personas particularmente significativas con relación al problema considerado en el proyecto, concentrándose más en un conjunto limitado de temas que en su trayectoria de vida.

En el contexto de la actividad de campo, después de una búsqueda bibliográfica y documental que intenta identificar y estudiar legislaciones, textos académicos, informativos o literarios relacionados con el tema de la investigación, es posible iniciar el contacto con los probables informantes. La forma más común de búsqueda de la colección bibliográfica y de la legislación sobre el tema estudiado es a través de Internet, recurriendo a las bibliotecas públicas o universidades.

La entrevista tiene una ventaja sobre otros instrumentos de investigación (cuestionarios u observaciones) que, en general, establecen una cierta jerarquía entre investigador y sujeto investigado. En el caso de Ho, a partir de un listado de preguntas prefijadas de acuerdo con los objetivos del proyecto, las entrevistas deben permitir divulgar múltiples experiencias. Y a pesar de que las entrevistas han estado presentes durante mucho tiempo en el desarrollo de las investigaciones cualitativas, en nuestro caso debemos reforzar una diferenciación ya dicha antes: la entrevista como técnica adoptada en Ho tiene fundamentos históricos, por lo que se pone gran cuidado en la selección de fuentes históricas. Su desarrollo puede yacer en documentos escritos y fotografías cuyo acceso sería notablemente más difícil, quizá imposible de otra forma. Además, ha de señalarse que se puede determinar un trabajo en términos de Ho si se reconoce su intención y ciertos procedimientos de retorno público de los resultados ${ }^{15}$.

El guion de la entrevista se puede modificar en el curso de las entrevistas, mediante la adición o eliminación de preguntas o su adaptación. Uno de los criterios para la selección de las personas para las entrevistas puede ser una red: un participante sugiere la participación de otro, que a su vez sugerirá nuevos informantes. En el momento de la grabación de la conversación, se deben tener algunos cuidados sencillos pero importantes para una buena entrevista y evitar sorpresas como: en contacto con el entrevistado, explicar brevemente los objetivos de la investigación y proporcionar el script previamente para que ayudarle a recordar las informaciones; durante la entrevista, presentar «activadores de la memoria» (fotografías, documentos, objetos, etc.); elegir un ambiente no ruidoso y confortable para el declarante (su casa o trabajo); realizar las entrevistas con hora fijada para el final no es muy productivos; el aparato de grabación (de audio o video) debe ser verificado antes y durante la entrevista.

Para el tratamiento de las informaciones obtenidas durante las entrevistas, el investigador inicialmente prepara un texto escrito que corresponde a la transcripción, en la forma más fiel posible del material grabado. En una segunda etapa, la transcripción, se pasa por el proceso llamado por muchos de «textualización»: con un cambio más radical en el texto de la transcripción, mediante la omisión de

is Meiny, J. C. S. B.: Manual de História Oral, São Paulo, Loyola, 2005. 
HISTORIA ORAL Y ANÁLISIS NARRATIVO DE NARRATIVAS EN LA INVESTIGACIÓN EN LA EDUCACIÓN MATEMÁTICA BRASILEÑA FERNANDO GUEDES CURY

algunas palabras, como los vicios del lenguaje y la reconstrucción del texto para una lectura más fluida.

La textualización, en general, se puede considerar como un texto cuyos autores son el entrevistado y el investigador, aunque muchas veces, como se puede confirmar por los estudios apuntados anteriormente, es un texto del investigador para el que el declarante autoriza (o no) lo que se ha dicho o escrito. En algunos casos las textualizaciones pueden presentarse como un diálogo, manteniendo las preguntas que se respondieron durante la grabación de entrevistas. Esta elección permite tener en cuenta que gran parte de la información dada por los entrevistados surge a partir de las preguntas realizadas por el investigador, lo que demuestra, por ejemplo, la importancia que debe darse a la entrevista, que requiere una cautelosa preparación previa del investigador.

La búsqueda de información sobre cada entrevistado y de su actuación en el contexto estudiado son elementos importantes para los investigadores que recurren a este tipo de trabajo. Pero señalamos que, entre los trabajos desarrollados utilizando Ho, la opción de presentar textualizaciones no es única o estandarizada, y se pueden encontrar diferentes formas de textualización de los distintos relatos disponibles (existen, por ejemplo, los investigadores que optan por un estilo de escritura en el que sólo el declarante se pronuncia en un texto junto a un monólogo). Este cuidado de la entrevista y la preparación de textualización es importante en el trabajo porque se determinó que las textualizaciones son parte importante de la narrativa histórica buscada por los investigadores.

Los aspectos éticos involucrados en el proceso de investigación son de suma importancia para garantizar el acceso público a un documento histórico. Así, después de que el entrevistado confirme las informaciones dadas en las entrevistas, la narrativa con la versión final (a disposición en el texto académico), el narrador generalmente firma un documento con una autorización (grabada en audio o video) para el uso académico de los registros.

De esta manera, se construyen narrativas a partir de relatos y este ejercicio sería uno de los principales fundamentos de Ho. Para la autora una situación vivida por el entrevistado no puede ser transmitida a otros sin ser narrada. Entonces, el narrador «se constituye en narrador» en el momento de la entrevista. Los relatos pueden producir, pues, un «conocimiento narrativo» próximo al conocimiento popular y muy usado en la construcción de biografías, narrativas que expresan intenciones, acciones e historias particulares. Este es un conocimiento de carácter temporal y particular y que representa significados y voces de los actores e investigadores.

La discusión sobre el interés en las ciencias sociales de visiones particulares/ personales del mundo se aproxima al desarrollo de lo que se ha denominado «método biográfico», que considera que cada individuo manifiesta de forma singular en sus acciones toda la estructura social a la que pertenece ${ }^{16}$. O sea, la construcción

16 Goldberg, M.: A Arte de Pesquisar: como fazer pesquisa qualitativa em ciências sociais, Rio de Janeiro, Record, 2003. 
o el estudio de una narración se constituye como un interesante medio de reunión social desde la especificidad irreductible de una vida individual.

En el próximo apartado hablaremos más sobre las narrativas y trataremos de la creación de estas no sólo como fuente de investigación histórica, sino como una alternativa para su análisis.

\section{Las narrativas como formas de producción de significados}

Narrar es contar una historia, narrarse es contar nuestra historia o una historia de lo que somos, fuimos o nos sentimos personajes. Es importante tener en cuenta que la narración siempre se realiza hacia alguien. Y creemos que la narración ofrece una posición de uno frente al otro. Además, las narrativas ofrecen la posibilidad de un análisis, pues concebimos el análisis como un proceso cíclico de producción de significados que se inicia cuando el oyente/lector/espectador de un texto se apropia de este texto de alguna manera tejiendo significados que son suyos, aunque haya sido producido de forma compartida con el autor del texto y construye una trama narrativa que va a ser escuchada/leída/vista por un tercero, que, a su vez, vuelve al principio del proceso al producir sus propios significados. En cada relato, por lo tanto, participan en su formación el autor y el oyente. El que narra, lo hace siempre hacia los demás. Y estos hilan y tejen, como lo diría el filosofo Walter Benjamin, participando en la narración y manteniéndose más o menos interesados, dando significado con gestos, miradas, énfasis, abreviaturas... Así, los primeros oyentes pueden llegar a ser los segundos narradores que escucharán la historia e irán a (re)contarla desde los significados producidos.

Entonces, intentar comprender una realidad, a través de cualquier tipo de análisis, teniendo en cuenta los informes, narraciones, en visiones, versiones sobre un hecho particular, hábitos y prácticas, incluye (o puede incluir) la comprensión de los modos de narrarlo otros: las formas en que el narrador asigna significado a sus propias experiencias. Sin embargo, podría plantearse una cuestión: ¿es realmente posible percibir las prácticas y experiencias de un narrador? ¿Prácticas y experiencias son susceptibles de ser narradas?

En primer lugar, en un intento por diferenciar la experiencia de una "práctica», esta no debe ser entendida como una forma de conocimiento inferior, considerada sólo como un punto de partida para el conocimiento o, incluso, como un obstáculo al «conocimiento verdadero $»^{17}$. Con la intención de legitimar la experiencia, Larrosa señala algunas precauciones necesarias: en primer lugar, en las investigaciones en Educación deberíamos eliminar de la palabra «experiencia» su connotación empírica, es decir, apartarla de la idea de "experimento». A continuación deberíamos eliminar todo dogmatismo, toda pretensión de autoridad y también separar experiencia de práctica. Eso es «pensar la experiencia no desde la acción

${ }^{17}$ Larrosa, J.: «Algunas notas sobre la experiencia y sus lenguajes», en Larrosa, J. R.L.L. (org.): Trajetórias e perspectivas da formação de educadores, São Paulo, UNESP, 2005, p. 470. 
HISTORIA ORAL Y ANÁLISIS NARRATIVO DE NARRATIVAS EN LA INVESTIGACIÓN EN LA EDUCACIÓN MATEMÁTICA BRASILEÑA FERNANDO GUEDES CURY

sino desde la pasión, desde una reflexión del sujeto sobre sí mismo desde el punto de vista de la pasión ${ }^{18}$. Por último, deberíamos evitar la tentación de hacer de la experiencia un concepto y simplemente tomarla como «modo de habitar el mundo de un ser que existe, de un ser que no tiene otro ser, otra esencia, que su propia existencia: corporal, finita, encarnada, en el tiempo y en el espacio, con otros» ${ }^{19}$.

Así, en muestra concepción historiográfica, un historiador debe explicar los episodios con los que se trata y no caracterizarlos como modelos en el mundo; puede incorporar las prácticas del cronista, sobre todo de los representantes clásicos (los cronistas medievales). Estos son los precursores de la historiografía moderna: el historiador, por tanto, debe promover el encuentro de los hilos históricos con los pequeños detalles de la vida cotidiana ${ }^{20}$.

Y llegamos, entonces, a una comprensión de lo que llamamos un análisis a través de las narrativas. La propuesta, debido a múltiples versiones presentadas en una investigación con narrativas, es trabajar con cada una de ellas, ya que cada una por sí misma es incompleta, considerándolas como modos de los narradores de narrarse a sí mismos y constituir sus verdades como sujetos, aceptando siempre una distinción entre lo que se vive y lo que es narrado. El análisis narrativo constituiría los significados de las experiencias de los narradores por la búsqueda de elementos unificadores y de develación de sujetos individuales e idiosincrasias de los contextos investigados, con el fin de expresar de modo auténtico la vida individual, sin manipular la voz de los participantes ${ }^{21}$.

Y con la intención de reflexionar sobre una posibilidad de análisis narrativo, es fiable decir que hay fundamentalmente dos modos de funcionamiento cognitivo, cada uno proporciona diferentes modos de planificación, construcción de la realidad de la experiencia. El primero es el modo paradigmático (o lógico-científico) que intenta construir un sistema formal de descripción y explicación. Este modo emplea la categorización en la conceptualización y las operaciones en que se establecen, se idealizan y se relacionan las categorías para formar una afirmación. El modo paradigmático lidia con la creación de causas genéricas y hace uso de procedimientos para asegurar la comprobación de las referencias y probar una veracidad empírica. Su forma de expresión está regulada por una necesidad de coherencia y no contradicción, y su dominio está definido por elementos observables -cuyas afirmaciones básicas refieren- y son conducidas por hipótesis prefijadas. Sin embargo, el modo narrativo asume que las acciones humanas son únicas e irrepetibles: su riqueza de matices no se puede mostrar en direcciones, categorías o proposiciones ${ }^{22}$.

8 LARROSA (2005: 47I).

19 LARRosa (2005: 472).

${ }_{20}$ Benjamin, W.: «O Narrador: considerações sobre a obra de Nikolai Leskov», en Benjamin, W.: Magia e Técnica, Arte e Política: ensaios sobre a literatura e a bistória da cultura, São Paulo, Brasiliense, 1994, pp. 197-221.

${ }^{21}$ Bolívar, A. B.: «De nobis ipsis silemus? Epistemología de la investigación biográfico-narrativa en educación», Revista Eletrónica de Investigación Educativa, vol. II, n. I (2002), pp. I-26.

22 Bruner, J.: Atos de Significação, Porto Alegre, Artes Médicas, 1997. 
Un análisis paradigmático de los datos narrativos consiste, por lo tanto, en un estudio de las narrativas a través de su categorización para llegar a generalizaciones del grupo estudiado que buscan, en estas narrativas, temas comunes. El análisis narrativo (de narrativas) pone el énfasis en la consideración de casos particulares. El producto de este análisis aparece como una nueva narrativa, la explicación de una trama o argumentos que hacen que los datos sean significativos, no en busca de elementos comunes, sino buscando un punto culminante de ellas único y que, en definitiva, no aspira a la generalización. El papel del investigador en este segundo tipo de análisis consiste en configurar los datos en una historia que los une y les da significado con la intención de mostrar el auténtico modo de vida individual sin recurrir a la voz de cada narrador. La trama puede ser construida temporal o temáticamente, pero lo importante es que permita comprender por qué sucedió algo. La propuesta es revelar el carácter único de un caso individual y proporcionar una comprensión de su particular complejidad o idiosincrasia ${ }^{23}$.

Algunas posibilidades de análisis narrativo de narrativas en la investigación educativa se están ejerciendo por grupos de investigación brasileños que usan la HO en la educación matemática. Entre todos los trabajos realizados -algunos ya mencionados anteriormente- hablaremos un poco más sobre los que han ejercitado ese modo de análisis. Los objetivos que han guiado estas investigaciones están vinculados a la historia de la educación matemática brasileña, pues intentan comprender los procesos de formación de maestros y las prácticas de enseñanza en diferentes contextos y temporalidades.

En una investigación sobre la formación de los primeros cursos para maestros de matemáticas del estado del Tocantins (Brasil) hemos presentado un relato (entre muchos otros posibles) sobre la creación de cursos de formación de maestros de matemáticas y formación de las instituciones de aquel estado ${ }^{24}$. Este presenta sus comprensiones desde los datos, de las circunstancias vividas y de cómo las historias de diferentes personas, apuntadas en sus testimonios, le ayudarán a entender un escenario específico. Para la preparación de tal narrativa el autor buscó en narrativas grabadas por él, textos, documentos y en su propia experiencia los puntos de convergencia y divergencia, cosas comunes y singulares, para componer una narración que puede ser entendida como núcleo de la investigación participada. La narrativa presentada en este trabajo es una creación, una interpretación, tejida de declaraciones y otros documentos recogidos durante la investigación. No debe ser entendida como un resumen, sino como un (re)significado de historias oídas, leídas, vistas, experimentadas durante la investigación.

Así, la narrativa se produce desde un movimiento de recuperación, persiguiendo pistas y rastros como lo hace un cazador cuando recrea los pasos de su presa para seguirla, uniendo piezas y, a pesar de las incertidumbres, se van explicitando,

${ }^{23}$ Bolívar, A. B.: «De nobis ipsis silemus? Epistemología de la investigación biográfico-narrativa en educación», Revista Eletrónica de Investigación Educativa, vol. II, n. I (2002), pp. I-26.

24 Cury, F. G.: Uma História da Formação de Professores de Matemática e das Instituições Formadoras do Estado do Tocantins, Tesis doctoral, Universidade Estadual Paulista, Rio Claro/ Brasil, 20II. 
en lo posible, las relaciones cambiantes de un tiempo y situación geográfica específicos, o sea, un contexto de posibilidades históricamente determinadas ${ }^{25}$. Los vínculos entre historia y narración conectan directamente la historia a otras formas de inteligibilidad de la realidad: el historiador, como lo hizo el cazador primitivo, debe aprender a captar pistas, rastros, a veces efímeros, y debe construir los hilos para tejerla. Así, la propuesta de la investigación es que el análisis narrativo intente escapar del dualismo causa-efecto que mira el mundo de forma lineal. La narrativa está conscientemente contaminada con sus percepciones, diciendo lo que fue posible decir, lo que ha sido considerado significativo, incluyendo toda la trayectoria.

En otro estudio ${ }^{26}$, se realizó el tratamiento y la teorización sobre narrativa, en una investigación con Ho, en la que se pretendió analizar el proceso de constitución de la identidad del Centro de Educación Matemática (CEM). Este ha sido un grupo que trabajó principalmente entre los años de 1984 a 1997 en la ciudad de São Paulo (Brasil) haciendo servicios de asesoramiento y consultoría especializada en educación matemática para las escuelas, juntas y directorios gubernamentales de educación.

En este trabajo, la «identidad» fue concebida en el sentido de los procesos de producción de significados $-\mathrm{O}$ «invenciones», o «expresiones del mundo real»para los agentes personales, o colectivos o de cosas, constituidos por discursos basados en un atributo cultural; o, incluso, un conjunto de atributos culturales interrelacionados que tienen prevalencia sobre otras fuentes de sentido. Guiados por esta «desconcepción» de identidad, se han creado y presentado diferentes procesos de producción de significados sobre el CEM, es decir, diferentes identidades de ese grupo. Para ello, fueron registrados y analizados quince testimonios orales , diez de los cuales procedentes de miembros de ese grupo, llamados «fragmentos de memoria». Como segundo objetivo de la investigación, Silva intentó constituir distintas teorizaciones sobre la identidad del grupo investigado con el fin de presentar los diferentes significados de los procesos de producción del grupo desde una mirada externa a él. Esas teorías, que se presentan en cuatro fragmentos, se elaboraron, respectivamente, sobre la base de René Descartes (fragmento XI); Émile Durkheim, George Herbert Mead, Peter Berger y Thomas Luckmann y, sobre todo, Norbert Elias (Fragmento XII); Etienne Wenger (Fragmento XIII) y Michel Foucault (Fragmento XIV). Silva consideró razonable abarcar tres puntos de vista que difieren principalmente por el lugar desde el cual se conforma el sujeto en el mundo: en su «interior» (perspectiva cartesiana), en la mezcla de su «interior» con el mundo cultural «exterior» (perspectiva sociológica) y las formas en que se representa el mundo cultural (perspectiva posmoderna).

Las narrativas -como resultado de la textualizaciones de las entrevistas- hablan sobre las historias de los informantes y sobre el grupo, y tienen como materia prima la memoria. Esos discursos narrativos son, así, los lugares donde se

25 Ginzburg, C.: Mitos, emblemas, indicios: morfología e historia, Barcelona, Gedisa, 2008.

26 Silva, H.: Centro de Educação Matemática: fragmentos de identidade, Tesis doctoral, Universidade Estadual Paulista, Rio Claro/Brasil, 2007. 
constituyen las primeras identidades del grupo CEM. De estas narraciones y referencias teóricas surgieron diferentes discursos científicos, es decir, modos de producir significados para «el establecimiento de la identidad del CEM» desde el punto de vista del conocimiento científico y, entre ellos, se establecieron nuevas identidades para este grupo.

Con el fin de cubrir todos los aspectos, incluso lingüísticos, de las diferentes teorías de la identidad en el análisis de las narrativas sobre el CEM, el texto se ha configurado bajo los moldes del análisis narrativo, pero de acuerdo con los cuatro discursos científicos diferentes. Se han producido textos literario-ficticios inspirados en el trabajo de heterónimos de Fernando Pessoa y situados en fragmentos aislados, cada uno de ellos en forma de diálogo narrativo entre la investigadora y un personaje heterónimo de ella («inventando» nuevos informantes de la investigación). El contenido de cada uno de estos fragmentos está asociado al análisis del proceso de constitución de las identidades del CEM, bajo las bases teóricas aceptadas y vividas por el personaje en cuestión. La idea general era que cada personaje ayudara a la investigadora en este proceso para entre todos hacer una «lectura plausible» de los parámetros teóricos adoptados.

La estrategia de heteronomía ayudó a señalar que los aspectos de la identidad son determinados exclusivamente por la forma como se ve este concepto y la situación en que las personas están en el momento que hablan sobre la identidad.

Un último ejemplo: la tesis doctoral titulada Projetos Minerva: caixa de jogos caleidoscópica, de Thiago Pedro Pinto ${ }^{27}$, es, como su título indica, una obra que más se asemeja a una caja que contiene varios juegos separados, casi independientes, por lo que el lector es quien elige cuál va empezar, seguir o terminar jugando/ leyendo. El Proyecto Minerva fue creado por el Gobierno federal brasileño para ofertar un curso complementario de enseñanza primaria y secundaria. Se trasmitió vía radio con un alcance a nivel nacional, en las décadas de 1970 y 1980. El autor intentó, con esta metáfora de los juegos, indicar multiplicidades de formas de aprehensión de los temas que implican el Proyecto Minerva, o mejor, Proyectos Minerva. El objetivo de la investigación, dice Pinto, fue producir tantos Minerva como fuera posible, teniendo en cuenta la multitud de fuentes que fue consultada: fuentes escritas (periódicos, informes, folletos, guiones, etc.), fuentes de comunicación (grabaciones de radio) y testimonios orales (entrevistas).

\section{APRESENTAÇÃO}

Esta é uma caixa de muitos jogos organizados ao longo do desenvolvimento de um projeto de pesquisa de doutorado. Cada jogo consiste de um sistema de símbolos linguísticos e imagens que visam a propiciar ao jogador a construção de significados para a expressão PROJETO MINERVA. Todos os jogos desta caixa foram elaborados para se jogar em grupo. Caso esteja sozinho, o jogador poderá criar outros jogadores a partir dos jogos aqui apresentados.

${ }^{27}$ Pinto, T. P.: Projetos Minerva: caixa de jogos caleidoscópica, Tesis doctoral, Universidade Estadual Paulista, Bauru/Brasil, 2013. 


\section{COMPONENTES}

Esta caixa de jogos é composta de 9 jogos apresentados separadamente em seus suportes físicos. No entanto, é importante alertar aos jogadores que alguns dos jogos podem ser jogados juntos, enquanto outros podem ser separados para formar outros tantos jogos. A própria caixa como um todo pode ser entendida, também, como um jogo. Sugere-se aos jogadores que, a partir destes jogos, criem outros e inventem, para eles, novas regras.

\section{PREPARAÇÃO}

a) Acomode-se confortavelmente para a leitura (sugere-se uma mesa para apoiar os textos ou uma poltrona com braços). b) Pegue material para fazer anotações. Elas podem ser necessárias.

\section{INÍCIO DO JOGO}

Este jogo não tem início. Ele já começou, assim que o jogador teve conhecimento desta caixa de jogos suas produções já começaram. Especificamente para os jogos aqui apresentados, sugerimos que você leia atentamente estas instruções, os apêndices e notas de rodapé que compõem os textos e esta caixa de jogos. Todo esse material pode tanto ser respeitado como subvertido.

\section{COMO JOGAR}

a) Distribua os jogos na mesa ou folheie-os para escolher um deles.

b) A partir deles, construa seus Projetos Minerva. Em cada um deles há notas de rodapé para auxiliá-los nesta construção.

c) Crie e recrie personagens, acredite no que julgar plausível -crie plausibilidades-, questione o que não acreditar. Compare as regras de cada jogo. Às vezes pode ser necessário abandonar algumas para jogar um outro jogo, às vezes será necessário retomá-las.

\section{COMO CONTAR OS PONTOS}

Os pontos que cada jogador ganha são contados de forma subjetiva: não há ganhador ou perdedor. Quanto mais diferenças ou semelhanças encontrar entre os jogos mais pontos terá. O jogador estabelece o que é «diferença» e o que é «semelhança», por mais que as regras e o jogo queiram pré-definir esses termos.

\section{TÉRMINO DO JOGO}

Quando quiser poderá fechar os jogos e abandoná-los. No entanto, ainda assim, o jogo não terminará. Ele poderá ser retomado sempre que o jogador desejar com ou sem o auxílio dos jogos desta caixa ${ }^{28}$.

Así se inicia el volumen Instrucciones de Uso, que muestra una forma de indicar al lector tanto la metáfora de la tesis (juegos para jugar, en el sentido literal del juego de palabras), como sugerencias sobre sus fundamentos teóricos y metodológicos, inspirados en el filósofo Ludwig Wittgenstein y sus juegos de lenguaje.

28 Pinto, 20I3: 9-Io. 
En el mismo volumen, pero en Apêndice A: jugos e caleidoscópios, el autor justifica la metáfora de los juegos caleidoscópicos señalando que la imagen del Proyecto Minerva puede ser construida y reconstruida por el lector de la misma manera que un caleidoscopio adopta una multitud de imágenes, construyéndolas y «deconstruyéndolas» con cada movimiento del juguete formado por espejos.

De los nueve volúmenes presentados, cuatro son de relatos de entrevistas realizadas por el investigador con profesores y estudiantes involucrados en el Proyecto. Son relatos que muestran asuntos políticos acerca de la creación y mantenimiento del Minerva; el trabajo involucrado en la producción de materiales y organización de los cursos; dinámica de sus clases; las dificultades de acceso a estudios por la gente de las comunidades más distantes de los centros más importantes del país, además de aspectos sociales, políticos y económicos que involucran estas comunidades.

En uno de los volúmenes, el autor muestra varios extractos sobre el Minerva buscando con ellos motivar a los lectores a formar sus propios proyectos $\mathrm{Mi}$ nerva usando los juegos de lenguaje, bajo la inspiración de Wittgenstein. En el volumen $A$ coleção da SOARMEC, por ejemplo, el autor hace una descripción de su búsqueda en el archivo de la Sociedad de Amigos y Oyentes de la Radio del Ministerio de Educación (SOARMEC), cuyo resultado es un minucioso catálogo y un mapa del archivo.

Para hablar sobre las «radioclases» de matemáticas, Pinto escribió el volumen de Produção de Radioaulas no Projeto Minerva, en que hay una descripción de una clase de matemáticas (sobre rectas y circunferencias) y descripciones de los materiales producidos en el texto que fue enviado a los estudiantes y los producidos por la radio. Este volumen discute las matemáticas producidas en diversos materiales (escritos y orales), que son diferentes, según Pinto, pues son lenguajes distintos -aquí se presenta nuevamente la concepción de juegos de lenguaje-.

En $A$ radio debate sobre o Projeto Minerva, Pinto produce un texto ficticio sobre un programa de radio llamado $A$ voz do povo para presentar aspectos del proyecto Minerva realizado a partir de los informes de sus informantes, documentos y análisis realizados en el desarrollo de la investigación. La intención del autor fue, como él mismo dijo, crear una foto compuesta, es decir, un texto ficticio de características estructurales similares a los relatos de los entrevistados durante la investigación y otros documentos consultados. Los debates del programa de radio creado dan la increíble impresión de que realmente estuvieran sucediendo, sugiriendo así el potencial de la estrategia de ficción para expresar casos ejemplares de lo que, aunque no sucedió, su contenido viene de expresiones sobre lo real (testimonios y documentos).

\section{Consideraciones finales}

En este texto planteamos que, a partir de las propuestas para la Historia desarrolladas especialmente en el siglo pasado para el análisis de nuevas fuentes, la 
HISTORIA ORAL Y ANÁLISIS NARRATIVO DE NARRATIVAS EN LA INVESTIGACIÓN EN LA EDUCACIÓN MATEMÁTICA BRASILEÑA FERNANDO GUEDES CURY

Ho surgió como una alternativa viable a la historiografía: escribir historias desde la experiencia acumulada por una persona como la principal fuente de investigación. El trabajo con Ho recurre fundamentalmente a la memoria y otros conceptos tales como: retención, recuerdo, olvido, selección, resentimiento, euforia, entusiasmos. Como metodología de investigación en la educación matemática brasileña, usa supuestos teóricos en relación directa con procedimientos prácticos buscando el apoyo de teorías diversas y el diálogo con diferentes áreas, tales como la Filosofía y la Sociología. Los trabajos desarrollados por algunos grupos de investigación en aquel país permitieron la elaboración de fuentes históricas así como resaltar distintas lecturas históricas, «verdades» plurales y coexistentes, incluso si se aplican a proyectos no históricos.

Señalamos que, en la parte práctica, el testimonio oral presenta el núcleo de la investigación, permitiendo exponer trayectorias individuales, eventos o procesos que en algunos casos no se pueden mirar de otra forma; además estos documentos orales son documentos generados para posibles investigaciones futuras. Así, en una investigación de Ho debemos seleccionar temas o personas, realizar entrevistas y hacer el tratamiento del producto que se puede analizar en relación a sus objetivos. Tales análisis deben considerar que en las encuestas de HO nos encontramos esencialmente apoyados por puntos de vista individuales, es decir, la subjetividad es un punto importante, y que tanto la narración cuanto la forma de construcción y organización de los discursos deben ser valoradas por el historiador.

Por último, presentamos ejemplos de estudios y análisis que hicieron uso de los llamados análisis narrativos de narrativas. En el paso del estudio de los documentos (incluso orales) hacia la composición de la escritura del discurso narrativo analítico, el historiador puede emplear las mismas estrategias de lenguaje utilizado por figuración de escritores de ficción y, en este sentido, el contenido del relato histórico se caracteriza por «invención». La historia, como todos sabemos, es también un conjunto de discursos llenos de significados (narrativas), estos forman una trama textual por la cual «tenemos acceso» a un pasado. No podemos apartarnos de esas formas narrativas para entender nuestro "mundo real». Para el estadunidense Hayden White, la única cosa que distingue los discursos «históricos» de los «de ficción» son, en esencia, los contenidos, en lugar de la forma de presentarlos, porque el contenido de los relatos históricos se refiere a hechos reales, cosas que realmente ocurrieron y eventos no imaginarios, inventados por el narrador ${ }^{29}$.

En el análisis narrativo de narrativas el énfasis está en la consideración de casos particulares y el producto de este análisis puede ser una (o más) narrativa (temporal o temática) que aspira a la explicación de una trama o argumentos que hacen de los datos que sean significativos - esto incluye, como hemos dicho, narrativas ficticias como modos de producción de significados de las historias que se desean contar-.

29 White, H.: Trópicos do discurso: ensaios sobre a crítica da cultura, São Paulo, Edusp, 1994. 


\section{Bibliografía}

Alberti, V:: «Narrativas pregnantes como jogos de linguagem: possibilidades da história oral à luz da teoria da linguagem de Wittgenstein», História Oral, Rio de Janeiro, v. II (2008), pp. I27-I48.

Amado, J. y Ferreira, M. M.: Usos e Abusos da Historia Oral, Rio de Janeiro, Fundação Getúlio Vargas, 1996.

Benjamin, W.: «O Narrador: considerações sobre a obra de Nikolai Leskov», en Benjamin, W.: Magia e Técnica, Arte e Politica: ensaios sobre a literatura e a bistória da cultura, São Paulo, Brasiliense, 1994, pp. 197-221.

Bolívar, A. B.: «De nobis ipsis silemus? Epistemología de la investigación biográfico-narrativa en educación», Revista Eletrónica de Investigación Educativa, vol. II, n. I (2002), pp. I-26.

Bruner, J.: Atos de Significação, Porto Alegre, Artes Médicas, 1997.

Cury, F. G.: Uma História da Formação de Professores de Matemática e das Instituições Formadoras do Estado do Tocantins, Tesis doctoral, Universidade Estadual Paulista, Rio Claro/Brasil, 2oII.

Garnica, A. V. M.; Fernandes, D. N. y Silva, H.: «Entre a amnésia e a vontade de nada esquecer: notas sobre Regimes de Historicidade e História Oral», Bolema, Rio Claro, v. 25 (20II), pp. 2I3-250.

Ginzburg, C.: Mitos, emblemas, indicios: morfología e historia, Barcelona, Gedisa, 2008.

Goldberg, M.: A Arte de Pesquisar: como fazer pesquisa qualitativa em ciências sociais, Rio de Janeiro, Record, 2003.

Hernández Díaz, J. M.: «Recensión: Vidigal, L.: “Os testemunhos oráis na escola. Historia oral e projectos pedagógicos”, Porto, Ediçoes Asa, I996». História de la Educación, vol. I6 (1997), pp. 647-648.

LARrosa, J.: «Algunas notas sobre la experiencia y sus lenguajes», en LARrosa, J. R. L. L. (org.): Trajetórias e perspectivas da formação de educadores, São Paulo, unEsP, 2005, p. 470.

MARquès, S.: «Importancia de la investigación oral para el estudio de la historia de la escuela franquista», Historia de la Educación, v. I2-I3 (1993-1994), pp. 435-447.

Meıнy, J. C. S. B.: Manual de História Oral, São Paulo, Loyola, 2005.

Pinto, T. P.: Projetos Minerva: caixa de jogos caleidoscópica, Tesis doctoral, Universidade Estadual Paulista, Bauru/Brasil, 2013.

Silva, H.: Centro de Educação Matemática: fragmentos de identidade, Tesis doctoral, Universidade Estadual Paulista, Rio Claro/Brasil, 2007.

Thompson, P.: A voz do passado: história oral, São Paulo, Paz e Terra, 1998.

White, H.: Trópicos do discurso: ensaios sobre a crítica da cultura, São Paulo, Edusp, 1994.

Nota final. Quería agradecer a la profesora María Teresa Gonzáles Astudillo (USAL) y al profesor Fiaban Arley Posada Balvin (UFRN) por las sugerencias y correcciones linguísticas del español. 